\title{
Treatment of Choledocholithiasis following Roux-en-Y Hepaticojejunostomy Using Double-Balloon Endoscopy
}

\author{
T.W.Spahn W. Grosse-Thie P.Spies M.K. Mueller \\ Department of General Internal Medicine/Gastroenterology, Marienhospital Osnabrück, \\ Academic Teaching Hospital of the University of Hannover Medical School, Osnabrück, Germany
}

Dear Sir,

Endoscopic treatment of gallstones in patients with a Roux-en-Y hepaticojejunostomy is frequently impaired, as the lengths of the remaining stomach and of the afferent loop exceed the length of the endoscope. Here we report the case of a 34-year-old female patient who had previously undergone Roux-en-Y hepaticojeju- nostomy and was referred to our hospital with choledocholithiasis. On admission, the jaundiced patient complained about abominal pain. Gallstones in intra- and extrahepatic bile ducts were detected by ultrasound and magnetic resonance cholangiography (fig. 1A). As the diameter of the gallstones exceeded the diameter of the hepaticojejunal anastomosis, dilation of the anastomosis using a balloon catheter was required prior to endoscopic extraction of the stones. Percutanenous transhepatic cholangiography performed in order to insert the dilation balloon revealed several gallstones in the common bile duct and in both branches of the he-
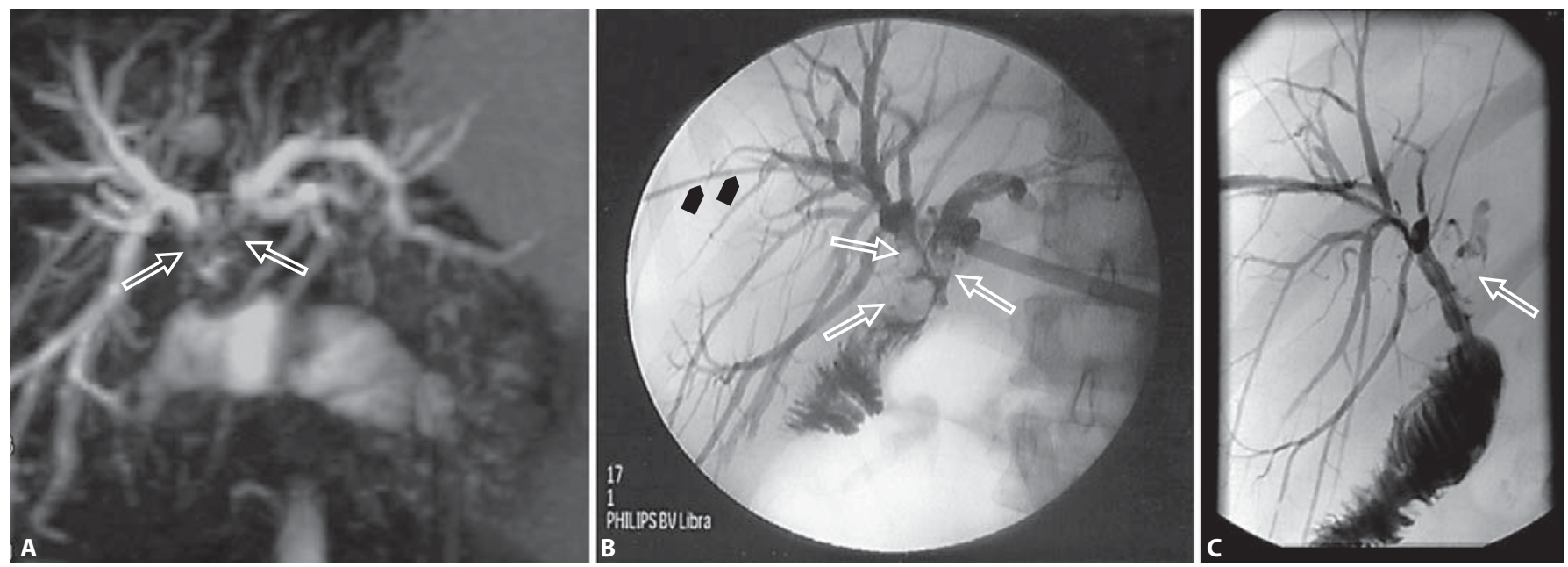

Fig. 1. A $\mathrm{T}_{2}$-weighted magnetic resonance cholangiography showing distended bile ducts and central occlusion of the central biliary system by multiple bile stones (arrows). B Percutaneous transhepatic cholangiography with drainage catheter (dark arrows) showing multiple gallstones in the common hepatic duct and intrahepatic lithiasis (open arrows). C Percutaneous transhepatic cholangiography showing occlusion of the left hepatic duct by remaining bile stones (arrow).

\section{KARGER}

Fax +41613061234 E-Mail karger@karger.ch www.karger.com
Thomas W. Spahn, MD

Department of General Internal Medicine/Gastroenterology

Marienhospital Osnabrück, Johannisfreiheit 2-4

DE-49074 Osnabrück (Germany)

Tel. +49541326 8105, Fax +49541326 4656,E-Mail thomas.spahn@mho.de 

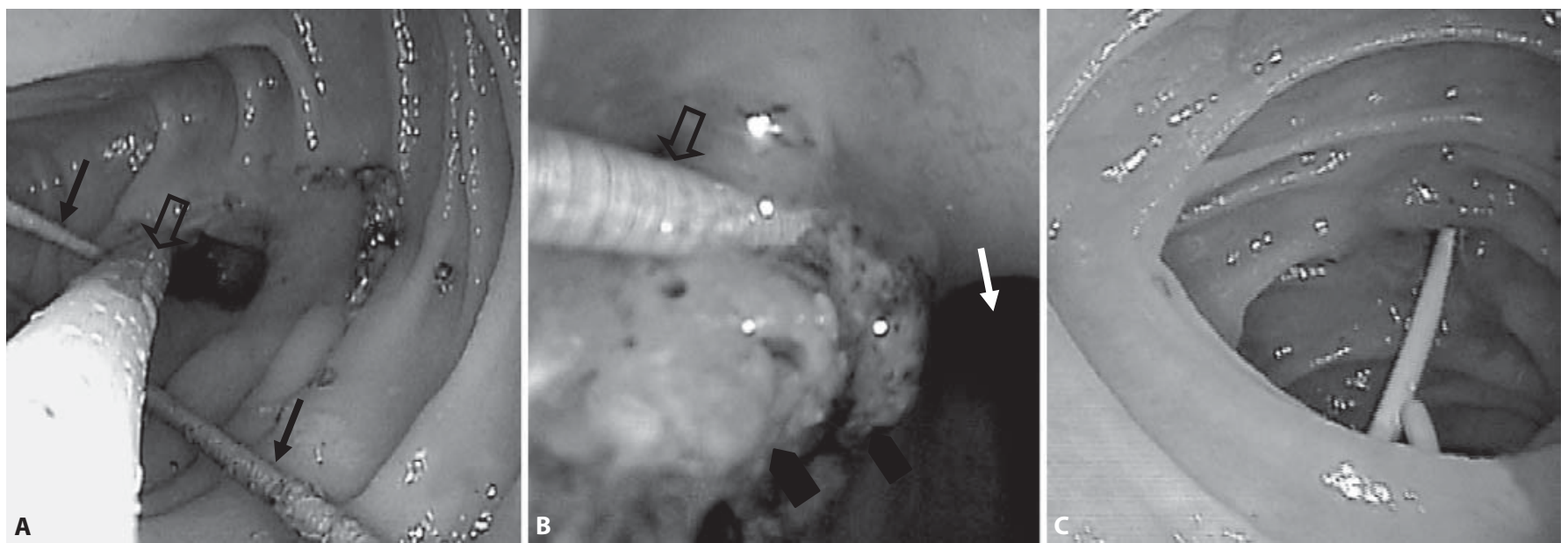

Fig. 2. Endoscopic extraction of the remaining gall stones. A A guide wire (dark arrows) is placed in the right and a Dormia basket in the left hepatic duct (open arrow). B Recanalized left hepatic duct (white arrow), Dormia basket (open arrow), and extracted gallstones (dark arrows). C Hepaticojejunostomy with internal-external drainage catheter.

patic ducts (fig. 1B). Even after dilation of the anastomosis the gallstones could only be removed from the common bile duct and the right biliary system. There were remaining gallstones in the left biliary tree on subsequent control cholangiography after placement of the biliary drainage catheter (fig. 1C). In order to save the patient from the potential complications of a left-sided percutaneous transhepatic cholangiography, double-balloon endoscopy (DBE) of the afferent loop was performed. Endoscopic inspection of common bile duct and both hepatic ducts revealed multiple gallstones in the left hepatic duct (fig. 2). All of the stones were subsequently endoscopically removed using a Dormia basket. An internal-external catheter was placed, and cholestasis subsequently normalized.

\section{Discussion}

The Roux-en-Y limb is a diversionary reconstruction usually applied for biliary disease or in liver transplantation, when the native bile duct cannot be used to cre- ate a duct-to-duct anastomosis. The intact stomach and a long distal duodenojejunal limb promote looping of the endoscope, making it technically more difficult to endoscopically reach the biliary-enteric anastomosis [1]. DBE has been established as an invaluable tool for diagnosis and treatment of small-bowel disease. Because of the length and the improved maneuverability of the endoscope, DBE has also been used for diagnostic and interventional indications in patients with the Roux-en-Y setting [2].The treatment of intrahepatic lithiasis in the setting of a Roux-en-Y limb comprises the combined use of endoscopic and percutaneous techniques. We first chose to perform the transcutaneous transhepatic approach in our patient, as this method is well established in our department. Our case demonstrates that $\mathrm{DBE}$ in combination with balloon dilation of the anastomosis of a hepaticojejunostomy allows to safely extract gallstones in a patient with a Rouxen-Y anastomosis. As the balloon dilation of an anastomotic stricture of a choledochojejunostomy has been described using the DBE technique [3], future patients with choledocholithiasis in a Roux-en-Y setting might primarily be treated by endoscopic dilation and extraction of gallstones, as this procedure does not carry the risks of a transcutaneous transhepatic approach.

\section{References}

1 Hintze RE, Adler A, Veltzke W, Abou-Rebyeh $\mathrm{H}$ : Endoscopic access to the papilla of Vater for endoscopic retrograde cholangiopancreatography in patients with Billroth II or Roux-en-Y gastrojejunostomy. Endoscopy 1997;29:69-73.

2 Kuno A, Yamamoto H, Kita H, Sunada K, Yano T, Hayashi Y, Sato H, Miyata T, Sekine Y, Iwamoto M, Ido K, Sugano K: Double-balloon enteroscopy through a Roux-en-Y anastomosis for EMR of an early carcinoma in the afferent duodenal limb. Gastrointest Endosc 2004;60:1032-1034.

3 Haruta H, Yamamoto H, Mizuta K, Kita Y, Uno T, Egami S, Hishikawa S, Sugano K, Kawarasaki H: A case of successful enteroscopic balloon dilation for late anastomotic stricture of choledochojejunostomy after living donor liver transplantation. Liver Transpl 2005;11:1608-1610. 